\title{
Sufficient and Persistent Blood Pressure Reduction in the Final Long-Term Results From SYMPLICITY HTN-Japan - Safety and Efficacy of Renal Denervation at 3 Years -
}

\author{
Kazuomi Kario, MD; Eiichiro Yamamoto, MD; Hirofumi Tomita, MD; Takafumi Okura, MD; \\ Shigeru Saito, MD; Takafumi Ueno, MD; Daiki Yasuhara, BSc; Kazuyuki Shimada, MD \\ on behalf of the SYMPLICITY HTN-Japan Investigators
}

\begin{abstract}
Background: SYMPLICITY HTN-Japan is a prospective, randomized, controlled trial comparing renal denervation (RDN) with standard pharmacologic therapy for treatment of uncontrolled hypertension (HTN).

Methods and Results: Patients enrolled had uncontrolled HTN, defined as office systolic blood pressure (SBP) $\geq 160 \mathrm{mmHg}$ and 24-h ambulatory SBP $\geq 135 \mathrm{mmHg}$, on $\geq 3$ antihypertensive drugs of maximally tolerated dose for at least 6 weeks prior to enrollment. Randomization was $1: 1$ to RDN or maintenance of current medical therapy (control). Patients were followed every 6 months postrandomization for up to 36 months. There were 22 patients randomized to RDN and 19 to control, and 11 patients were crossed over and received RDN at 6 months post-randomization. For the RDN group $(n=22)$, office SBP reduction was $-32.8 \pm 20.1 \mathrm{mmHg}$ and office DBP reduction was $-15.8 \pm 12.6 \mathrm{mmHg}$ at 36 months post-procedure, both $\mathrm{P}<0.001$. For the combined RDN and crossover group $(n=33$ ), office SBP reduction was $-26.7 \pm 18.9 \mathrm{mmHg}$ and office DBP reduction was $-12.7 \pm 11.8 \mathrm{mmHg}$ at $30 \mathrm{months}$ postprocedure, both $\mathrm{P}<0.001$. There were no procedural-, device- or treatment-related safety events through 36 months.
\end{abstract}

Conclusions: SYMPLICITY HTN-Japan is the first randomized controlled trial to evaluate RDN in an Asian population. Despite the small number of enrollments, results show patients who received RDN therapy maintained SBP reduction out to 36 months.

Key Words: Ambulatory blood pressure; Asia; Renal denervation; Resistant hypertension

$\mathbf{T}$ he prevalence of hypertension (HTN) in the Japanese population is estimated to be at least 40 million cases, ${ }^{\mathbf{1 , 2}}$ with approximately $4 \%$ of patients labeled as having resistant HTN. ${ }^{3}$ Catheter-based renal denervation (RDN) has been used to treat resistant HTN in patients in previous clinical studies, with promising results, ${ }^{\mathbf{4} 6}$ although the randomized, sham-controlled SYMPLICITY HTN-3 study failed to demonstrate efficacy. ${ }^{7}$ However, 3 recent sham-controlled clinical studies demonstrating significant blood pressure (BP) reductions after RDN alone or in the presence of antihypertensive drug therapy have renewed interest in this technique. . $-10^{-10}$

SYMPLICITY HTN-Japan (HTN-J) is the first randomized controlled trial to assess the efficacy of RDN in an Asian population. The trial was stopped prematurely after the SYMPLICITY HTN-3 results were reported, and hence the small enrollment in HTN-J substantially reduced the power of the study. ${ }^{11}$ The current report presents 3-year safety and efficacy data from HTN-J.

\begin{abstract}
Methods
Study Design

HTN-J is a prospective, randomized, controlled clinical trial designed to evaluate the safety and efficacy of RDN compared with standard of care medical therapy. Patients with uncontrolled HTN were enrolled and randomized 1:1 to RDN or control. Control patients had the option of crossing over and receiving RDN after 6 months. The trial was approved by each center's institutional review board and was conducted according to the Declaration of Helsinki. All patients signed written informed consent to participate in the trial. The trial is registered at www.clinicaltrials.gov (NCT01644604).
\end{abstract}

Received September 10, 2018; revised manuscript received November 20, 2018; accepted January 8, 2019; J-STAGE Advance Publication released online February 13, 2019 Time for primary review: 29 days

Division of Cardiovascular Medicine, Jichi Medical University School of Medicine, Tochigi (K.K.); Kumamoto University Hospital, Kumamoto (E.Y.); Hirosaki University School of Medicine and Hospital, Aomori (H.T.); Ehime University Hospital, Ehime (T.O.); Shonan Kamakura General Hospital, Kanagawa (S.S.); Kurume University Hospital, Fukuoka (T.U.); Medtronic Japan, Tokyo (D.Y.); and New Oyama Municipal Hospital, Tochigi (K.S.), Japan

Mailing address: Kazuomi Kario, MD, Professor and Chairman, Division of Cardiovascular Medicine, Jichi Medical University School of Medicine, 3311-1 Yakushiji, Shimotsuke, Tochigi 329-0498, Japan. E-mail: kkario@jichi.ac.jp

ISSN-1346-9843 All rights are reserved to the Japanese Circulation Society. For permissions, please e-mail: cj@j-circ.or.jp 


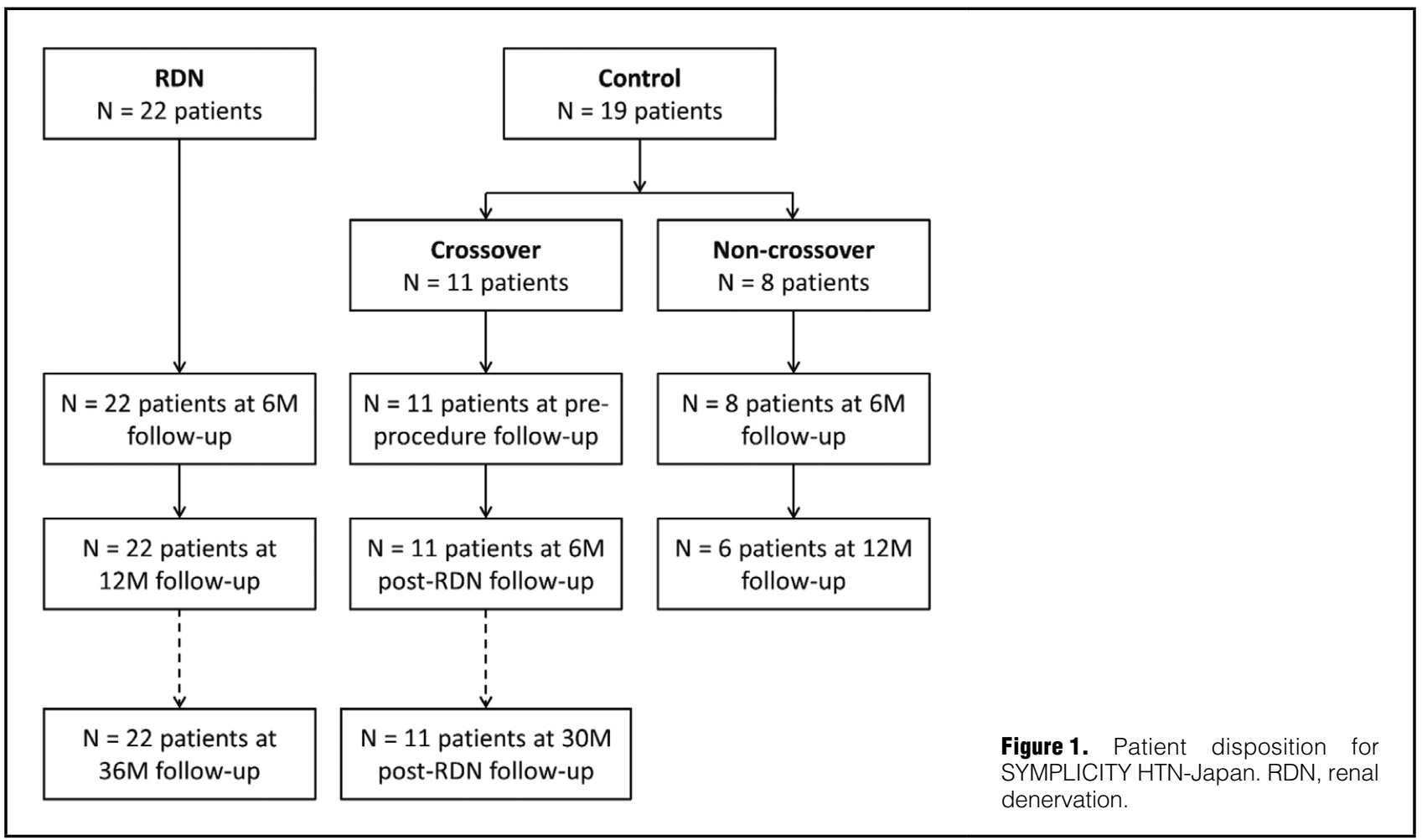

Table 1. Baseline Characteristics and Blood Pressure Measurements

\begin{tabular}{lcc} 
& $\begin{array}{c}\text { Renal denervation } \\
(\mathbf{n = 2 2})\end{array}$ & $\begin{array}{c}\text { Crossover } \\
(\mathbf{n = 1 1 )}\end{array}$ \\
Age (years) & $59.5 \pm 11.9$ & $55.2 \pm 13.9$ \\
Male & $68.2 \%(15)$ & $72.7 \%(8)$ \\
BMI $\left(\mathrm{kg} / \mathrm{m}^{2}\right)$ & $27.0 \pm 5.5$ & $28.7 \pm 4.9$ \\
Renal insufficiency (eGFR $\left.<60 \mathrm{~mL} / \mathrm{min} / 1.73 \mathrm{~m}^{2}\right)$ & $4.5 \%(1)$ & $9.1 \%(1)$ \\
Known renal artery stenosis & $0.0 \%(0)$ & $0.0 \%(0)$ \\
Obstructive sleep apnea & $9.1 \%(2)$ & $9.1 \%(1)$ \\
Stroke & $13.6 \%(3)$ & $9.1 \%(1)$ \\
Type 2 diabetes & $36.4 \%(8)$ & $63.6 \%(7)$ \\
Hospitalization for hypertensive crisis & $0.0 \%(0)$ & $9.1 \%(1)$ \\
Hypercholesterolemia & $27.3 \%(6)$ & $36.4 \%(4)$ \\
Current smoking & $31.8 \%(7)$ & $27.3 \%(3)$ \\
Office SBP & $181.0 \pm 18.0$ & $174.5 \pm 22.4$ \\
Office DBP & $92.4 \pm 16.2$ & $97.8 \pm 25.4$ \\
\hline
\end{tabular}

No significant differences between renal denervation and crossover groups. BMI, body mass index; DBP, diastolic blood pressure; SBP, systolic blood pressure.

\section{Patients}

Enrolled patients from 17 centers in Japan were at least 20 and up to 80 years old with uncontrolled HTN, defined as office systolic BP (SBP) $\geq 160 \mathrm{mmHg}$ and average 24-h SBP $\geq 135 \mathrm{mmHg}$ while on at least 3 antihypertensive medications at maximally tolerated doses, including a diuretic, for a minimum of 6 weeks prior to enrollment. Key exclusion criteria included estimated glomerular filtration rate $<45 \mathrm{~mL} / \mathrm{min} / 1.73 \mathrm{~m}^{2}$ (using the modified calculation method for Japanese patients ${ }^{12}$ ), type 1 diabetes mellitus and secondary causes of HTN.

After enrollment but prior to randomization, patients had 2 screening follow-up visits to ensure SBP was still within range. Patients were also required to keep a diary to record home BP measurements and antihypertensive medication use. In addition, enrolled patients underwent 24-h ambulatory BP monitoring (ABPM) and were required to have 24-h SBP $\geq 135 \mathrm{mmHg}$ to proceed to randomization.

Renal angiography was performed in all patients to confirm anatomic eligibility. Anatomic exclusions included main renal arteries with $<4 \mathrm{~mm}$ diameter or $<20 \mathrm{~mm}$ treatable length, the presence of renal accessory arteries, renal artery stenosis $(>50 \%)$ or a renal artery aneurysm. Patients with no anatomic exclusion criteria were randomized 1:1 


\begin{tabular}{|c|c|c|c|c|}
\hline & \multicolumn{2}{|c|}{$\begin{array}{l}\text { Renal denervation } \\
(\mathrm{n}=22)\end{array}$} & \multicolumn{2}{|c|}{$\begin{array}{l}\text { Renal denervation and crossover } \\
\qquad(n=33)\end{array}$} \\
\hline & 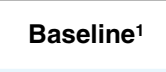 & $\begin{array}{l}30 \text { months } \\
\text { post-randomization }\end{array}$ & Baseline $^{1}$ & $\begin{array}{l}30 \text { months } \\
\text { post-randomization }\end{array}$ \\
\hline No. of antihypertensive medications & $4.9 \pm 1.5$ & $5.1 \pm 1.5$ & $5.0 \pm 1.7$ & $5.1 \pm 1.4$ \\
\hline Aldosterone antagonist & $45.5 \%(10)$ & $50.0 \%(11)$ & $45.5 \%(15)$ & $51.5 \%(17)$ \\
\hline$a-1$-blocker & $22.7 \%(5)$ & $45.5 \%(10)$ & $27.3 \%(9)$ & $45.5 \%(15)$ \\
\hline$a$-2-agonist & $0.0 \%(0)$ & $4.5 \%(1)$ & $0.0 \%(0)$ & $3.0 \%(1)$ \\
\hline Angiotensin-converting enzyme inhibitor & $9.1 \%(2)$ & $13.6 \%(3)$ & $12.1 \%(4)$ & $15.2 \%(5)$ \\
\hline Angiotensin-receptor blocker & $100.0 \%(22)$ & $86.4 \%(19)$ & $100.0 \%(33)$ & $90.9 \%(30)$ \\
\hline$\beta$-blocker & $81.8 \%(18)$ & $81.8 \%(18)$ & $81.8 \%(27)$ & $78.8 \%(26)$ \\
\hline Calcium-channel blocker & $95.5 \%(21)$ & $95.5 \%(21)$ & $93.9 \%(31)$ & $97.0 \%(32)$ \\
\hline Diuretic & $100.0 \%(22)$ & $95.5 \%(21)$ & $100.0 \%(33)$ & $90.9 \%(30)$ \\
\hline Direct renin inhibitor & $0.0 \%(0)$ & $0.0 \%(0)$ & $0.0 \%(0)$ & $0.0 \%(0)$ \\
\hline Vasodilator & $0.0 \%(0)$ & $0.0 \%(0)$ & $0.0 \%(0)$ & $0.0 \%(0)$ \\
\hline
\end{tabular}

${ }^{1}$ Baseline measurement for renal denervation patients at screening visit 2, baseline measurement for crossover patients at 6 months.

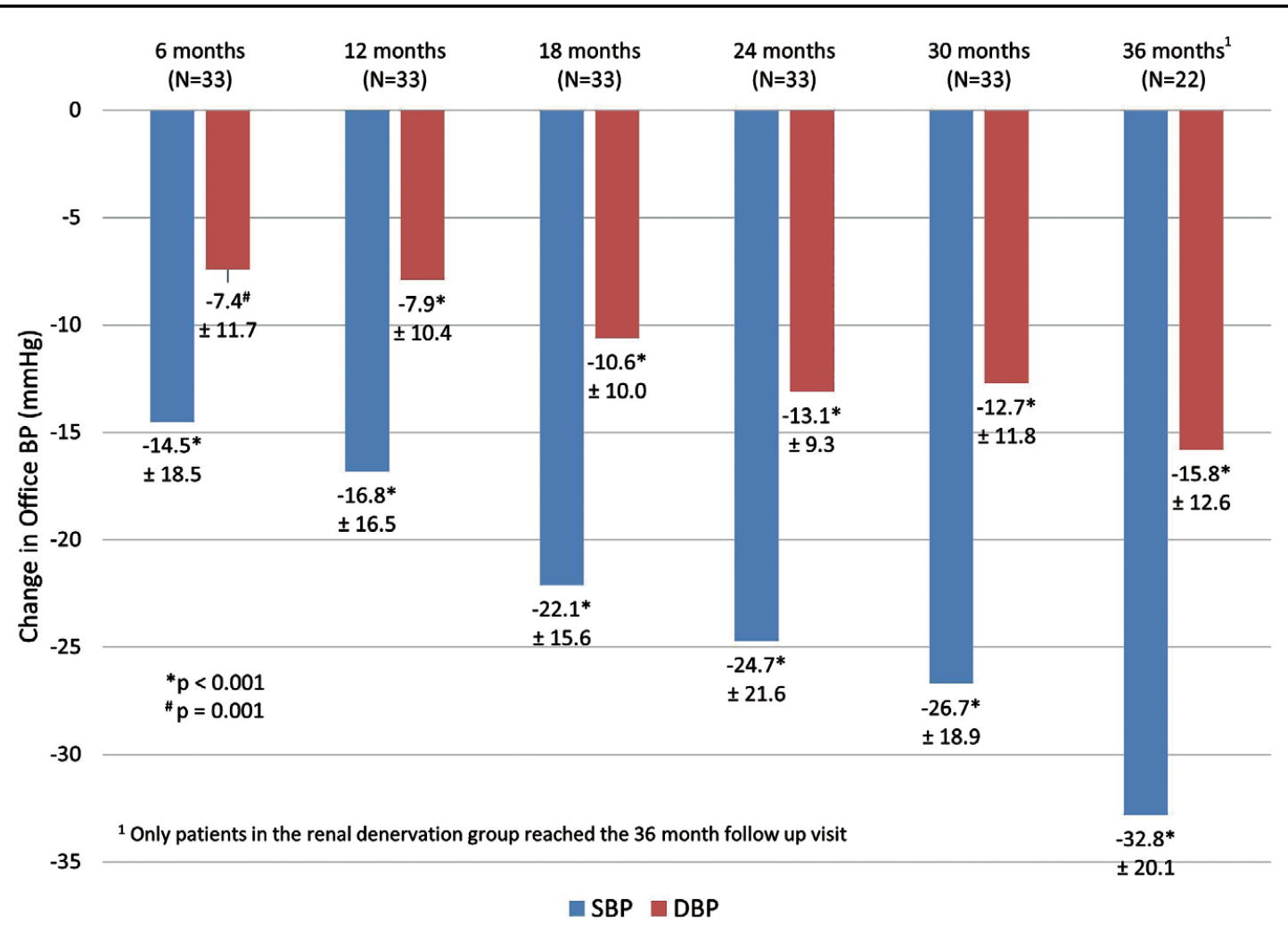

Figure 2. Reduction in office systolic (SBP) and diastolic (DBP) blood pressures from baseline through 36 months post-procedure for combined renal denervation and crossover patients. P-values compare BP measured at follow-up to baseline.

to the RDN procedure or maintenance of their established antihypertensive therapy (control group). Randomization was stratified by study center.

\section{RDN Procedure}

RDN was performed using the Symplicity ${ }^{\mathrm{TM}}$ generator and the mono-electrode Symplicity Flex ${ }^{\mathrm{TM}}$ radiofrequency catheter. Individual ablations were applied for $120 \mathrm{~s}$ each, with 4-6 ablations planned for each renal artery in a helical pattern. Patients in the control group were eligible for crossover to the RDN procedure after 6 months.

\section{Endpoints}

The primary efficacy endpoint was the change in office SBP 


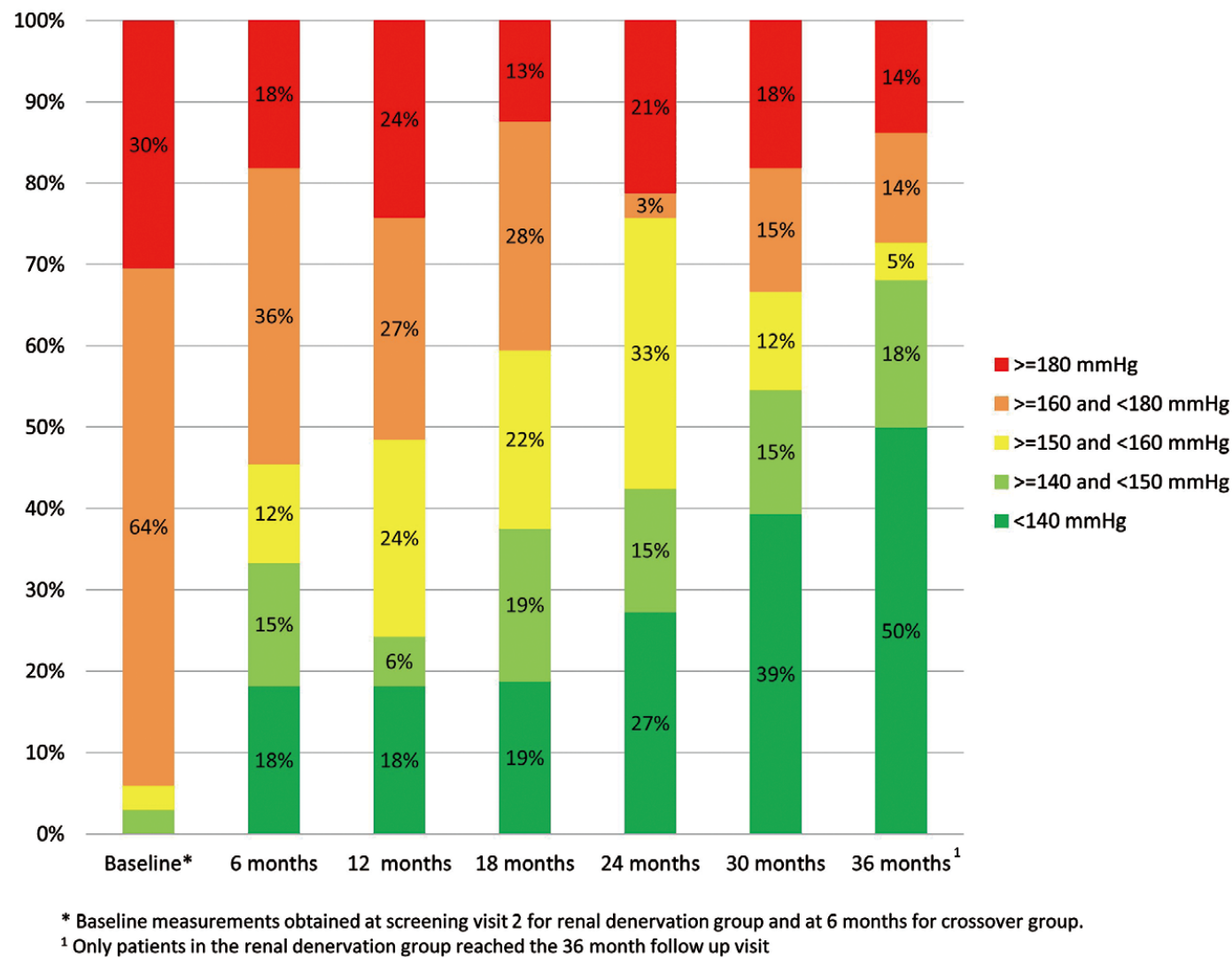

Figure 3. Distribution of office systolic blood pressure (SBP) measurements through 36 months for combined renal denervation and crossover patients.

from baseline to 6 months, and was also assessed at 12, 18, 24, 30 and 36 months post-procedure. RDN patients were followed out to 36 months post-procedure, but data were only collected up to 30 months post-procedure for crossover patients. Change in office diastolic BP (DBP) from baseline to every 6 months out to 36 months was a secondary efficacy endpoint. Hourly changes in ambulatory SBP and DBP from baseline to 6 months were also compared. The secondary safety endpoint was the composite of 1-month all-cause death, endstage renal disease, significant embolic event resulting in end-organ damage, renal artery dissection or perforation requiring intervention, vascular complications, hospitalization for hypertensive crisis or new renal artery stenosis $>70 \%$ confirmed on angiography within 6 months after randomization. Prescribed antihypertensive medications were recorded every 6 months.

\section{Statistical Analysis}

Analyses were performed according to the intent-totreat principle. Continuous variables are presented as mean \pm standard deviation and categorical variables are presented as number and percentage per treatment group. Between-group differences were compared using a 2-sample t-test or Fisher's exact test. Two-sided 95\% confidence intervals for the differences between treatment groups were also calculated. Differences in BP between baseline and follow-up were calculated using the paired t-test. Post hoc analyses compared mean hourly changes in ambulatory SBP. All data were analyzed using SAS version 9.2 or higher (SAS Institute, Cary, NC, USA).

\section{Results}

Baseline characteristics of enrolled patients have been previously presented.11 Patient disposition is shown in Figure 1. All RDN patients completed follow-up every 6 months through 36 months, and all crossover patients completed follow-up every 6 months to 30 months postRDN. For the 8 non-crossover patients, 1 was ineligible because of controlled BP and the other 7 were prohibited from receiving RDN after the HTN-3 results were announced. ${ }^{7}$ There were no significant differences in baseline SBP and DBP between RDN and crossover groups (Table 1).

Prescribed antihypertensive medication usage did not change significantly from preprocedure to 36 months postprocedure for the RDN group (Table 2). Prescribed $\alpha-1$ blocker usage increased from $27.3 \%$ to $45.5 \%$, while angiotensin-receptor blocker and diuretic usage both decreased slightly from $100 \%$ to $86.4 \%$ and $95.5 \%$, respectively. At baseline and 36 months, the majority of patients were prescribed a calcium-channel blocker and a $\beta$-blocker and no patients were prescribed a vasodilator or direct renin inhibitor. Similar trends are seen for the RDN+ 


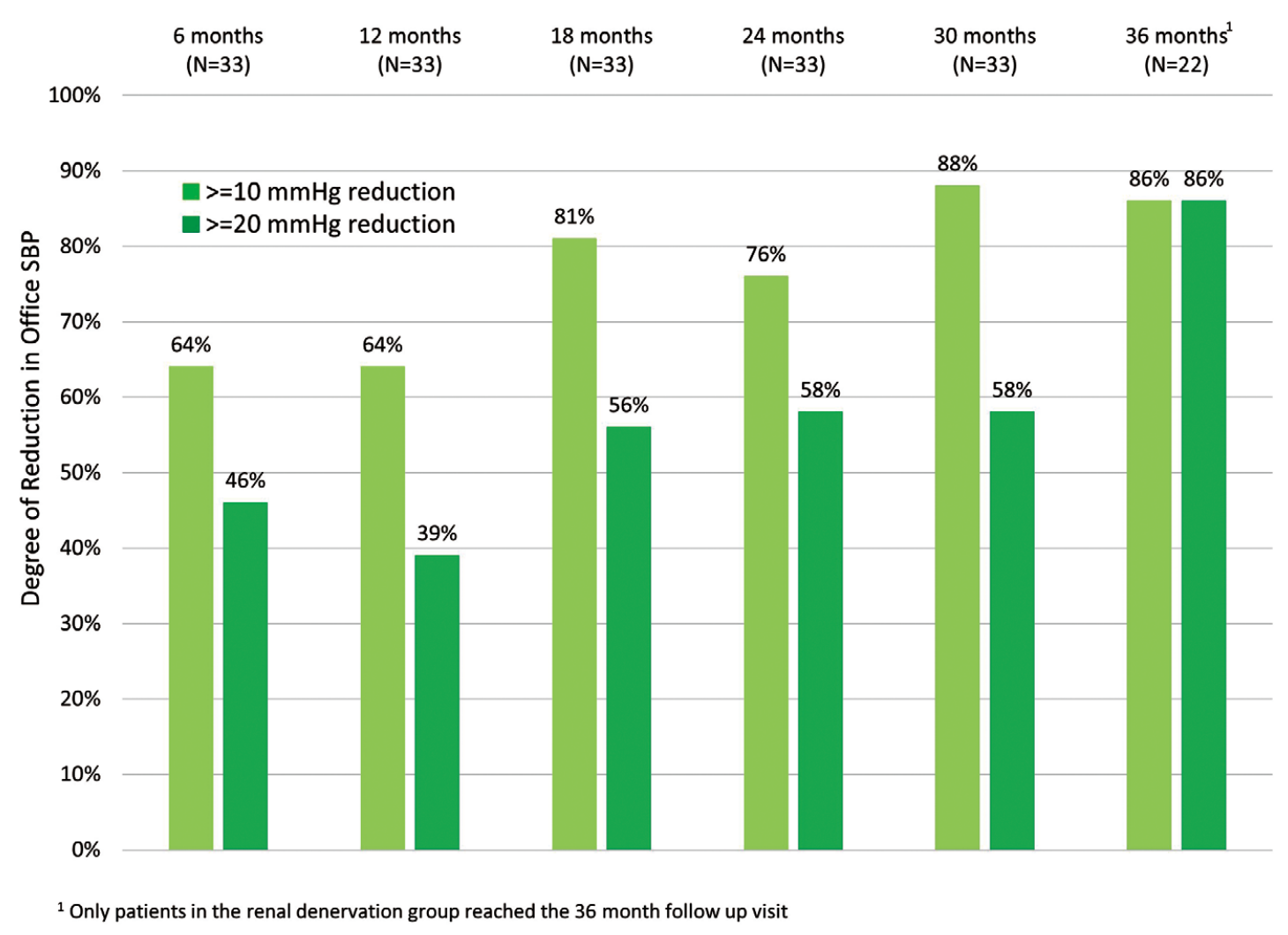

Figure 4. Percent of patients with a change in office systolic blood pressure (SBP) of 10 or $20 \mathrm{mmHg}$ for combined renal denervation and crossover patients.

crossover group at 30 months post-procedure (Table 2).

Reductions in office SBP and DBP were sustained out to 36 months post-procedure for RDN patients and 30 months post-procedure for crossover patients (Figure 2). BP measurements were not collected for non-crossover patients after 12 months post-randomization. Reduction at 36 months for the RDN group was $-32.8 \pm 20.1 \mathrm{mmHg}$ for office SBP and $-15.8 \pm 12.6 \mathrm{mmHg}$ for office DBP (both $\mathrm{P}<0.001$ vs. baseline). For the combined RDN+crossover treated patient group $(\mathrm{n}=33)$, SBP reduction was $-26.7 \pm 18.9 \mathrm{mmHg}$ and DBP reduction was $-12.7 \pm 11.8 \mathrm{mmHg}$ at 30 months post-procedure, both $\mathrm{P}<0.001$ for change from baseline. Individual patient responses to RDN at 30 months postprocedure and at 6 months post-randomization are shown in Supplementary Figure.

The distribution of office SBP measurements for treated patients is shown in Figure 3. The percent of patients with office SBP $<140 \mathrm{mmHg}$ increased from $18.2 \%$ at 6 months to $50 \%$ at 36 months. Reduction in office SBP by 10 and $20 \mathrm{mmHg}$ for treated patients is illustrated in Figure 4. The percent of patients with at least a $20-\mathrm{mmHg}$ drop in office SBP increased from $46 \%$ at 6 months to $86 \%$ at 36 months.

Hourly changes in ambulatory SBP (Figure 5A) from baseline to 6 months post-procedure for the RDN and crossover patients showed reductions throughout most of the day with minimal changes from baseline to 6 months post-randomization in the control group (Figure 5B).

As previously reported, at 6 months post-procedure, there were no major adverse events in any patient. ${ }^{11}$ This included death, new-onset endstage renal disease, embolic event resulting in end-organ damage, renal artery reintervention, vascular complication requires surgical repair, and hospitalization for hypertensive crisis. At 36 months, the rate remained zero for these events for all randomized patients. No patients experienced a myocardial infarction in either group, but $2 \mathrm{RDN}$ patients had a stroke (Supplementary Table).

\section{Discussion}

Analysis of HTN-J patients at 36 months demonstrated statistically significant and sustained reductions from baseline in office SBP $(-32.8 \mathrm{mmHg})$ and DBP $(-15.8 \mathrm{mmHg})$ for patients post-RDN. All the RDN and crossover patients maintained follow-up every 6 months through 36 months post-randomization. Furthermore, there were no procedural-, device-, or treatment-safety events related to RDN recorded out to 36 months post-randomization in any treated patient. Despite the fact that patients in HTN-J had resistant uncontrolled HTN with prior history of stroke (18\%), type 2 diabetes $(51 \%)$ and other comorbidities, ${ }^{11}$ the only serious adverse events were 2 patients who experienced an ischemic stroke: 1 patient had a cardioembolic stroke more than 2 years post-randomization with a history of smoking, cerebral hemorrhage, peripheral artery disease, coronary artery disease, and diabetes treated with 


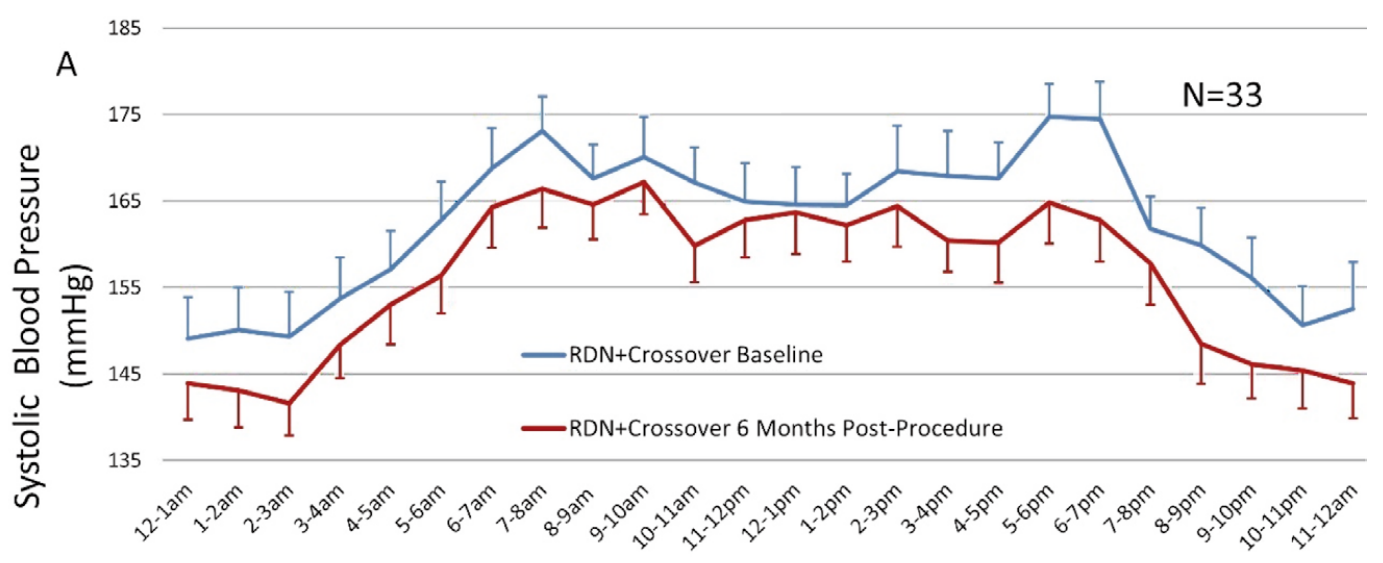

B $^{185}$

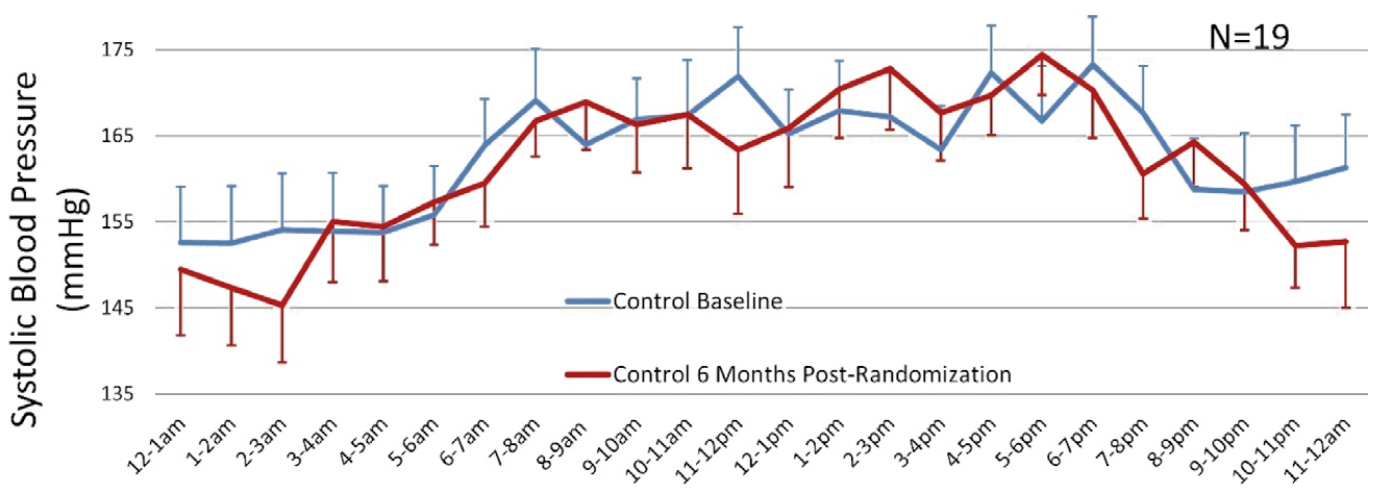

Figure 5. Hourly changes in systolic blood pressure (SBP) for (A) renal denervation and crossover patients from baseline to 6 months post-procedure and (B) control patients from baseline to 6 months post-randomization. Error bars represent one standard error.

insulin; the second was a former smoker with history of obstructive sleep apnea, abdominal aortic aneurysm treated by EVAR repair, angina pectoris and had a cerebral infarction more than 1 year after randomization. Both strokes, which occurred in these high-risk patients with polyvascular disease, were adjudicated to be unrelated to the device, procedure or therapy by the independent Clinical Events Committee.

Limited long-term follow-up data have been reported on the efficacy of RDN. The sustained BP reductions seen in HTN-J are similar to 3-year SBP and DBP reductions in SYMPLICITY HTN-1 $(-32 \mathrm{mmHg} \text { and }-14 \mathrm{mmHg})^{5}$ and HTN-2 ( $-33 \mathrm{mmHg}$ and $-14 \mathrm{mmHg}) .{ }^{4} \mathrm{~A}$ small prospective study also using the Symplicity Flex ${ }^{\mathrm{TM}}$ catheter found 3-year $\mathrm{SBP} / \mathrm{DBP}$ reductions of $-29 /-14 \mathrm{mmHg}$ in patients with accessory renal arteries and $-25 /-15 \mathrm{mmHg}$ in patients without. ${ }^{13}$ The all-comers Global Symplicity Registry reported a smaller 3-year SBP decrease of $-19 \mathrm{mmHg}$, likely because patients enrolled were eligible to be treated with lower baseline BPs. ${ }^{14}$ Moreover, the BP reduction in HTN-J was greater at 24 and 36 months compared with 6 and 12 months. The mechanism for the continuous reduction in $\mathrm{BP}$ is unclear but has been reported in other studies ${ }^{5,13}$ and could be related to vascular remodeling or changes in renin-angiotensin-system activity.
The present results contrast with those of the Symplicity HTN-3 trial, which failed to show a significantly greater $\mathrm{BP}$ reduction following RDN as compared with sham control. ${ }^{7}$ Notably, the inclusion and exclusion criteria were similar for Symplicity HTN-J and Symplicity HTN-3, as both trials included patients with treatment-resistant HTN. Both trials also used the same unipolar RF ablation system (Symplicity Flex). The primary difference between the trials was that the control group in Symplicity HTN-J was not blinded to therapy as in HTN-3, which included a sham procedure. Recently, potential confounding factors were identified in the HTN-3 trial design that prevented the trial from meeting its primary endpoint, including variable medication adherence, procedural differences, and the patient population. ${ }^{15}$ Given the recent positive results from multiple sham-controlled trials, these confounding factors may have obscured the efficacy of the RDN procedure in HTN-3. In particular, the difference in results between HTN-J and HTN-3 may be related to better or perhaps more consistent adherence to prescribed antihypertensive drug regimens in HTN-J as compared with HTN-3, thereby allowing the treatment effect of RDN to be readily demonstrated. It is also possible that Asian patients may be better candidates for RDN because of a higher prevalence of sympathetically-mediated HTN as compared with 
US patients. This hypothesis is supported by a recent subanalysis of the ONTARGET trial population indicating worse nonpersistence with antihypertensive drug therapy among black and white patients as compared with an Asian sub-population. ${ }^{16}$

The importance of lowering BP for cardiovascular risk reduction has been well documented. ${ }^{17,18}$ At 30 months post-randomization, $88 \%$ of the RDN and crossover patients in HTN-J had at least a $10-\mathrm{mmHg}$ reduction in SBP (Figure 4). In a recent meta-analysis, SBP reduction $\geq 10 \mathrm{mmHg}$ corresponded to a $13 \%$ risk reduction in allcause death, a $27 \%$ risk reduction in stroke events, a $28 \%$ risk reduction in heart failure, and a $17 \%$ risk reduction in coronary artery disease. ${ }^{18}$ Office and not $24-\mathrm{h}$ ambulatory $\mathrm{BP}$ was measured long-term in HTN-J. Given that elevated early morning ${ }^{19}$ and nocturnal ${ }^{20} \mathrm{BP}$ have been shown to incur increased cardiovascular risks, long-term assessment of 24-h ambulatory BP post-RDN will be important for future clinical studies.21

Plotting mean hourly BP measurements supports the credibility of these findings by demonstrating the effect of this therapy throughout the $24-h$ daily cycle. Reductions in hourly BP after catheter-based RDN at 3 months in SPYRAL HTN-OFF MED ${ }^{22}$ showed the greatest changes in the night-time and early morning hours, whereas the 6-month SPYRAL HTN-ON MED ${ }^{10}$ trial demonstrated uniform reductions throughout $24 \mathrm{~h}$ following RDN. HTN-J showed reductions throughout most of the 24-h period for the patients who underwent RDN, similar to the other 2 studies. All 3 studies showed no difference in the control group's hourly BP tracings between baseline and follow-up. These findings are potentially important as they are different from the pattern observed following antihypertensive drug use where BP reduction is less at night-time or in the early morning prior to daily morning drug ingestion. ${ }^{23}$ These differences indicate that $\mathrm{RDN}$ effects are "always on" and may affect BP throughout the $24 \mathrm{~h}$ day, reducing BP at night and in the early morning when the risk of cardiovascular events is higher. ${ }^{24-26}$

Not only is HTN-J the first randomized controlled clinical trial for RDN in Asian patients, but the results from HTN-J may signify that long-term results from SPYRAL HTN-OFF and ON MED trials will also show continuous sustained BP reductions. The 6-month office SBP reduction of $-16.6 \mathrm{mmHg}$ in HTN-J led to progressive SBP reduction of more than $-30 \mathrm{mmHg}$ at 36 months. Even though HTN-J did not include a sham procedure, medication changes between baseline and 6 months were rare for both the RDN and control groups ( $9.1 \%$ vs. 5.3\%, respectively), ${ }^{11}$ limiting this confounding factor in the trial. Moreover, treatment differences between the RDN and control groups for 24-h SBP were similar for HTN-J and other recent sham-controlled RDN trials. ${ }^{8-10}$

Although antihypertensive medication adherence was not measured in HTN-J, previous RDN studies report $50-60 \%$ of patients were non-adherent to antihypertensive medications, despite known medication surveillance. ${ }^{10,27}$ Analysis of medication adherence in an Asian population (COMFORT) found a correlation between low adherence and poor BP control. ${ }^{28}$ The prevalence of uncontrolled sustained HTN in the Asian population is estimated to have increased from $36 \%$ to $58 \%$ using the new AHA/ACC 2017 definition, ${ }^{29}$ so the challenges of treating HTN are growing.

\section{Impact of RDN in Asia}

Stroke, especially hemorrhagic stroke, and non-ischemic heart failure are common outcomes of HTN-related cardiovascular disease in Asia, and the association between $\mathrm{BP}$ and cardiovascular disease is stronger in Asia than in the West. ${ }^{30}$ Thus, earlier, stricter 24-h BP control, including early morning and nocturnal BP control, is especially important for Asians. ${ }^{29,31}$ In addition, Asian patients with drug-resistant HTN have higher morning and nocturnal $\mathrm{BP}$ variability than Western patients, even when office BP is comparable. ${ }^{32}$ Considering these Asian characteristics, RDN and its persistent 24-h BP control could be effective in reducing cardiovascular events in Asian populations.

\section{Study Limitations}

HTN-J was limited by its small sample size caused by premature discontinuation of enrollment. The control group did not have a sham procedure, which may have influenced endpoint assessment. Control patients had the option to cross over at 6 months after randomization, so there was no active control group at 36 months. Medication adherence was not measured, and ABPM data were not collected long-term in the study.

\section{Conclusions}

HTN-J is the first randomized controlled trial to evaluate RDN in an Asian population. Despite small enrollment numbers, the study results showed sustained reductions in office SBP and DBP post-RDN to 36 months. The 24-h ABPM demonstrated BP reduction throughout $24 \mathrm{~h}$. RDN was safe - there were 2 strokes that were adjudicated as unrelated to the procedure, device, or therapy.

\section{Acknowledgments}

The authors gratefully acknowledge all of the HTN-Japan Coinvestigators; Kunihiro Yamagata, MD and Hidehiko Hara, MD, for safety consultancy; Martin Fahy, MS, and Minglei Liu, PhD, from Medtronic for statistical support; Beth Ferri, $\mathrm{PhD}$, for assistance with the manuscript; and Sandeep Brar, MD and Myra Fan for study support and expert review. This work was supported by Medtronic Japan (ClinicalTrials.gov NCT01644604). The institutions and primary investigators for the SYMPLICITY HTN-Japan study are listed in the Supplementary Appendix.

\section{Disclosures}

K.K. received research funding from Otsuka holdings Co. and honoraria from Terumo Corporation; H.T. is a concurrent professor in the endowed department (the Advanced Management of Cardiac Arrhythmias of Hirosaki University Graduate School of Medicine) by Medtronic Japan Co., Ltd.; S.S. serves as a proctor for EvolutR and Evolut Pro TAVI devices; D.Y. is an employee of Medtronic Japan. Other authors report no conflict of interests.

\section{References}

1. Miura K, Nagai M, Ohkubo T. Epidemiology of hypertension in Japan: Where are we now? Circ J 2013; 77: 2226-2231.

2. Ogihara T, Kikuchi K, Matsuoka H, Fujita T, Higaki J, Horiuchi M, et al. The Japanese Society of Hypertension Guidelines for the Management of Hypertension (JSH 2009). Hypertens Res 2009; 32: 3-107.

3. Kario K, Okura A, Okawara Y, Tomitani N, Ikemoto T, Hoshide S. Impact of introducing catheter-based renal denervation into Japan for hypertension management: Estimation of number of target patients and clinical relevance of ambulatory blood pressure reduction. Curr Hypertens Rev 2016; 12: 156-163.

4. Esler MD, Bohm M, Sievert H, Rump CL, Schmieder RE, Krum $\mathrm{H}$, et al. Catheter-based renal denervation for treatment of patients with treatment-resistant hypertension: 36 month results from the SYMPLICITY HTN-2 randomized clinical trial. Eur 
Heart J 2014; 35: $1752-1759$.

5. Krum H, Schlaich MP, Sobotka PA, Bohm M, Mahfoud F, Rocha-Singh K, et al. Percutaneous renal denervation in patients with treatment-resistant hypertension: Final 3-year report of the Symplicity HTN-1 study. Lancet 2014; 383: 622-629.

6. Osborn JW, Banek CT. Catheter-based renal nerve ablation as a novel hypertension therapy: Lost, and then found, in translation. Hypertension 2018; 71: 383-388

7. Bhatt DL, Kandzari DE, O'Neill WW, D'Agostino R, Flack JM, Katzen BT, et al. A controlled trial of renal denervation for resistant hypertension. N Engl J Med 2014; 370: 1393-1401.

8. Townsend RR, Mahfoud F, Kandzari DE, Kario K, Pocock S, Weber MA, et al. Catheter-based renal denervation in patients with uncontrolled hypertension in the absence of antihypertensive medications (SPYRAL HTN-OFF MED): A randomised, sham-controlled, proof-of-concept trial. Lancet 2017; 390: 2160 2170.

9. Azizi M, Schmieder RE, Mahfoud F, Weber MA, Daemen J, Davies J, et al. Endovascular ultrasound renal denervation to treat hypertension (RADIANCE-HTN SOLO): A multicentre, international, single-blind, randomised, sham-controlled trial. Lancet 2018; 391: 2335-2345.

10. Kandzari DE, Bohm M, Mahfoud F, Townsend RR, Weber MA, Pocock S, et al. Effect of renal denervation on blood pressure in the presence of antihypertensive drugs: 6-month efficacy and safety results from the SPYRAL HTN-ON MED proof-ofconcept randomised trial. Lancet 2018; 391: 2346-2355.

11. Kario K, Ogawa H, Okumura K, Okura T, Saito S, Ueno T, et al. SYMPLICITY HTN-Japan: First randomized controlled trial of catheter-based renal denervation in Asian patients. Circ $J$ 2015; 79: 1222-1229.

12. Shimamoto K, Ando K, Fujita T, Hasebe N, Higaki J, Horiuchi $\mathrm{M}$, et al. The Japanese Society of Hypertension Guidelines for the Management of Hypertension (JSH 2014). Hypertens Res 2014; 37: 253-390.

13. Bartus K, Podolec J, Sadowski J, Litwinowicz R, Zajdel W, Bartus M, et al. Blood pressure reduction in patients with accessory renal arteries and bilateral single renal arteries after catheter-based renal denervation: A prospective study with 3-year follow-up. Pol Arch Intern Med 2017; 127: 423-428.

14. Mahfoud F, Brilakis N, Bohm M, Narkiewicz K, Ruilope L, Schlaich M, et al. TCT-761: Long-term (3-year) safety and effectiveness from the Global SYMPLICITY Registry of renal denervation in a real world patient population with uncontrolled hypertension. J Am Coll Cardiol 2016; 68: B308.

15. Kandzari DE, Bhatt DL, Brar S, Devireddy CM, Esler M, Fahy M, et al. Predictors of blood pressure response in the SYMPLICITY HTN-3 trial. Eur Heart J 2015; 36: 219-227.

16. Bohm M, Schumacher H, Laufs U, Sleight P, Schmieder R, Unger $\mathrm{T}$, et al. Effects of nonpersistence with medication on outcomes in high-risk patients with cardiovascular disease. $\mathrm{Am}$ Heart J 2013; 166: 306-314.e307

17. Bundy JD, Li C, Stuchlik P, Bu X, Kelly TN, Mills KT, et al. Systolic blood pressure reduction and risk of cardiovascular disease and mortality: A systematic review and network metaanalysis. JAMA Cardiol 2017; 2: 775-781.

18. Ettehad D, Emdin CA, Kiran A, Anderson SG, Callender T, Emberson J, et al. Blood pressure lowering for prevention of cardiovascular disease and death: A systematic review and metaanalysis. Lancet 2016; 387: 957-967.

19. Kario K. Global impact of 2017 American Heart Association/ American College of Cardiology Hypertension Guidelines: A perspective from Japan. Circulation 2018; 137: 543-545.

20. Boggia J, Li Y, Thijs L, Hansen TW, Kikuya M, BjorklundBodegard K, et al. Prognostic accuracy of day versus night ambulatory blood pressure: A cohort study. Lancet 2007; 370: $1219-1229$.

21. Whelton PK, Carey RM, Aronow WS, Casey DE Jr, Collins KJ, Dennison Himmelfarb C, et al. 2017 ACC/AHA/AAPA/ABC/ ACPM/AGS/APhA/ASH/ASPC/NMA/PCNA Guideline for the Prevention, Detection, Evaluation, and Management of High Blood Pressure in Adults: A report of the American College of Cardiology/American Heart Association Task Force on Clinical Practice Guidelines. J Am Coll Cardiol 2018; 71: 2199-2269.

22. Kario K, Bohm M, Mahfoud F, Townsend R, Weber M, Patel $\mathrm{M}$, et al. Twenty-four-hour ambulatory blood pressure reduction patterns following renal denervation in the SPYRAL HTN-OFF MED trial. Circulation 2018; 138: 1602-1604.

23. Kario K. Essential manual of 24 hour blood pressure management: From morning to nocturnal hypertension. Chichester, UK: John Wiley \& Sons Inc., 2015.

24. Kario K. Nocturnal hypertension: New technology and evidence. Hypertension 2018; 71: 997-1009.

25. Kario K, Pickering TG, Umeda Y, Hoshide S, Hoshide Y, Morinari $\mathrm{M}$, et al. Morning surge in blood pressure as a predictor of silent and clinical cerebrovascular disease in elderly hypertensives: A prospective study. Circulation 2003; 107: 1401-1406.

26. Kario K, Tomitani N, Kanegae H, Yasui N, Nishizawa M, Fujiwara T, et al. Development of a new ICT-based multisensor blood pressure monitoring system for use in hemodynamic biomarker-initiated anticipation medicine for cardiovascular disease: The National IMPACT Program Project. Prog Cardiovasc Dis 2017; 60: 435-449.

27. Azizi M, Pereira H, Hamdidouche I, Gosse P, Monge M, Bobrie $\mathrm{G}$, et al. Adherence to antihypertensive treatment and the blood pressure-lowering effects of renal denervation in the Renal Denervation for Hypertension (DENERHTN) Trial. Circulation 2016; 134: 847-857.

28. Matsumura K, Arima H, Tominaga M, Ohtsubo T, Sasaguri T, Fujii K, et al. Impact of antihypertensive medication adherence on blood pressure control in hypertension: The COMFORT study. QJM 2013; 106: 909-914.

29. Kario K. Global Impact of 2017 American Heart Association/ American College of Cardiology Hypertension Guidelines: A perspective from Japan. Circulation 2018; 137: 543-545.

30. Kario K, Chen CH, Park S, Park CG, Hoshide S, Cheng HM, et al. Consensus document on improving hypertension management in Asian patients, taking into account Asian characteristics. Hypertension 2018; 71: 375-382.

31. Kario K, Wang JG. Could $130 / 80 \mathrm{mmHg}$ be adopted as the diagnostic threshold and management goal of hypertension in consideration of the characteristics of Asian populations? Hypertension 2018; 71: 979-984.

32. Kario K, Bhatt DL, Brar S, Bakris GL. Differences in dynamic diurnal blood pressure variability between Japanese and American treatment-resistant hypertensive populations. Circ $J$ 2017; 81: $1337-1345$.

\section{Supplementary Files}

Please find supplementary file(s);

http://dx.doi.org/10.1253/circj.CJ-18-1018 